\title{
Superstability of the functional equation with a cocycle related to distance measures
}

\author{
Young Whan Lee ${ }^{1}$ and Gwang Hui Kim²* \\ Dedicated to Professor Shih-sen Chang on the occasion of his 80th birthday
}

${ }^{\text {*Correspondence: }}$ ghkim@kangnam.ac.kr ${ }^{2}$ Department of Mathematics, Kangnam University, Yongin, Gyeonggi 446-702, Korea Full list of author information is available at the end of the article

\begin{abstract}
In this paper, we obtain the superstability of the functional equation $f(p r, q s)+f(p s, q r)=\theta(p q, r s) f(p, q) f(r, s)$ for all $p, q, r, s \in G$, where $G$ is an Abelian group, $f$ a functional on $G^{2}$, and $\theta$ a cocycle on $G^{2}$. This functional equation is a generalized form of the functional equation $f(p r, q s)+f(p s, q r)=f(p, q) f(r, s)$, which arises in the characterization of symmetrically compositive sum-form distance measures, and as products of some multiplicative functions. In reduction, they can be represented as exponential functional equations. Also we investigate the superstability with following functional equations: $f(p r, q s)+f(p s, q r)=\theta(p q, r s) f(p, q) g(r, s)$, $f(p r, q s)+f(p s, q r)=\theta(p q, r s) g(p, q) f(r, s), f(p r, q s)+f(p s, q r)=\theta(p q, r s) g(p, q) g(r, s)$, $f(p r, q s)+f(p s, q r)=\theta(p q, r s) g(p, q) h(r, s)$.
\end{abstract}

MSC: 39B82; 39B52

Keywords: distance measure; superstability; multiplicative function; stability of functional equation

\section{Introduction}

Let $(G, \cdot)$ be an Abelian group. Let $I$ denote the open unit interval $(0,1)$. Let $\mathbb{R}$ and $\mathbb{C}$ denote the set of real and complex numbers, respectively. Let $\mathbb{R}_{+}=\{x \in \mathbb{R} \mid x>0\}$ be a set of positive real numbers and $\mathbb{R}_{k}=\{x \in \mathbb{R} \mid x>k>0\}$ for some $k \in \mathbb{R}$.

Further, let

$$
\Gamma_{n}^{o}=\left\{P=\left(p_{1}, p_{2}, \ldots, p_{n}\right) \mid 0<p_{k}<1, \sum_{k=1}^{n} p_{k}=1\right\}
$$

denote the set of all $n$-ary discrete complete probability distributions (without zero probabilities), that is, $\Gamma_{n}^{o}$ is the class of discrete distributions on a finite set $\Omega$ of cardinality $n$ with $n \geq 2$. Over the years, many distance measures between discrete probability distributions have been proposed. The Hellinger coefficient, the Jeffreys distance, the Chernoff coefficient, the directed divergence, and its symmetrization $J$-divergence are examples of such measures (see [1] and [2]).

Almost all similarity, affinity or distance measures $\mu_{n}: \Gamma_{n}^{o} \times \Gamma_{n}^{o} \rightarrow \mathbb{R}_{+}$that have been proposed between two discrete probability distributions can be represented in the sum 
form

$$
\mu_{n}(P, Q)=\sum_{k=1}^{n} \phi\left(p_{k}, q_{k}\right)
$$

where $\phi: I \times I \rightarrow \mathbb{R}$ is a real-valued function on unit square, or a monotonic transformation of the right side of (1.1), that is,

$$
\mu_{n}(P, Q)=\psi\left(\sum_{k=1}^{n} \phi\left(p_{k}, q_{k}\right)\right)
$$

where $\psi: \mathbb{R} \rightarrow \mathbb{R}_{+}$is an increasing function on $\mathbb{R}$. The function $\phi$ is called a generating function. It is also referred to as the kernel of $\mu_{n}(P, Q)$.

In information theory, for $P$ and $Q$ in $\Gamma_{n}^{o}$, the symmetric divergence of degree $\alpha$ is defined as

$$
J_{n, \alpha}(P, Q)=\frac{1}{2^{\alpha-1}-1}\left[\sum_{k=1}^{n}\left(p_{k}^{\alpha} q_{k}^{1-\alpha}+p_{k}^{1-\alpha} q_{k}^{\alpha}\right)-2\right]
$$

It is easy to see that $J_{n, \alpha}(P, Q)$ is symmetric. That is, $J_{n, \alpha}(P, Q)=J_{n, \alpha}(Q, P)$ for all $P, Q \in \Gamma_{n}^{o}$. Moreover, it satisfies the composition law

$$
\begin{aligned}
& J_{n m, \alpha}(P * R, Q * S)+J_{n m, \alpha}(P * S, Q * R) \\
& \quad=2 J_{n, \alpha}(P, Q)+2 J_{m, \alpha}(R, S)+\lambda J_{n, \alpha}(P, Q) J_{m, \alpha}(R, S)
\end{aligned}
$$

for all $P, Q \in \Gamma_{n}^{o}$ and $R, S \in \Gamma_{m}^{o}$ where $\lambda=2^{\alpha-1}-1$ and

$$
P * R=\left(p_{1} r_{1}, p_{1} r_{2}, \ldots, p_{1} r_{m}, p_{2} r_{1}, \ldots, p_{2} r_{m}, \ldots, p_{n} r_{m}\right) .
$$

In view of this, symmetrically compositive statistical distance measures are defined as follows. A sequence of symmetric measures $\left\{\mu_{n}\right\}$ is said to be symmetrically compositive if for some $\lambda \in \mathbb{R}$,

$$
\begin{aligned}
& \mu_{n m}(P \star R, Q \star S)+\mu_{n m}(P \star S, Q \star R) \\
& \quad=2 \mu_{n}(P, Q)+2 \mu_{m}(R, S)+\lambda \mu_{n}(P, Q) \mu_{m}(R, S)
\end{aligned}
$$

for all $P, Q \in \Gamma_{n}^{o}, S, R \in \Gamma_{m}^{o}$, where

$$
P * R=\left(p_{1} r_{1}, p_{1} r_{2}, \ldots, p_{1} r_{m}, p_{2} r_{1}, \ldots, p_{2} r_{m}, \ldots, p_{n} r_{m}\right) .
$$

Chung, Kannappan, Ng and Sahoo [1] characterized symmetrically compositive sumform distance measures with a measurable generating function. The following functional equation:

(FE) $f(p r, q s)+f(p s, q r)=f(p, q) f(r, s)$ 
holding for all $p, q, r, s \in I$ was instrumental in the characterization of symmetrically compositive sum-form distance measures. They proved the following theorem giving the general solution of this functional equation $(F E)$.

Suppose $f: I^{2} \rightarrow \mathbb{R}$ satisfies the functional equation $(F E)$, that is,

$$
f(p r, q s)+f(p s, q r)=f(p, q) f(r, s)
$$

for all $p, q, r, s \in I$. Then

$$
f(p, q)=M_{1}(p) M_{2}(q)+M_{1}(q) M_{2}(p)
$$

where $M_{1}, M_{2}: \mathbb{R} \rightarrow \mathbb{C}$ are multiplicative functions. Further, either $M_{1}$ and $M_{2}$ are both real or $M_{2}$ is the complex conjugate of $M_{1}$. The converse is also true.

The stability of the functional equation $(F E)$, as well as the four generalizations of $(F E)$, namely,

$\left(F E_{f g}\right) f(p r, q s)+f(p s, q r)=f(p, q) g(r, s)$,

$\left(F E_{g f}\right) f(p r, q s)+f(p s, q r)=g(p, q) f(r, s)$,

$\left(F E_{g g}\right) f(p r, q s)+f(p s, q r)=g(p, q) g(r, s)$,

$\left(F E_{g h}\right) f(p r, q s)+f(p s, q r)=g(p, q) h(r, s)$

for all $p, q, r, s \in G$, were studied by Kim and Sahoo in $[3,4]$. For other functional equations similar to $(F E)$, the interested reader should refer to [5-8], and [9].

The present work continues the study for the stability of the Pexider type functional equation of $(F E)$ added a cocycle property to the conditions in the results $[3,4]$. These functional equations arise in the characterization of symmetrically compositive sum-form distance measures, products of some multiplicative functions. In reduction, they can be represented as a (hyperbolic) cosine (sine, trigonometric) functional equation, exponential, and Jensen functional equation, respectively.

Tabor [10] investigated the cocycle property. The definition of cocycle as follows:

Definition 1 A function $\theta: G^{2} \rightarrow \mathbb{R}$ is a cocycle if it satisfies the equation

$$
\theta(a, b c) \theta(b, c)=\theta(a b, c) \theta(a, b), \quad \forall a, b, c \in G
$$

For example, if $F(x, y)=\frac{f(x) f(y)}{f(x y)}$ for a function $f: \mathbb{R} \rightarrow \mathbb{R}_{+}$, then $F$ is a cocycle. Also if $\theta(x, y)=\ln (x) \ln (y)$ for a function $\theta: \mathbb{R}_{+}{ }^{2} \rightarrow(\mathbb{R},+)$, then $\theta$ is a cocycle, that is, $\theta(a, b c)+$ $\theta(b, c)=\theta(a b, c)+\theta(a, b)$, and in this case, it is well known that $\theta(x, y)$ is represented by $B(x, y)+M(x y)-M(x)-M(y)$ where $B$ is an arbitrary skew-symmetric biadditive function and $M$ is some function [11]. If $\theta(x, y)=a^{\ln (x) \ln (y)}$, then $\theta: \mathbb{R}_{+}^{2} \rightarrow(\mathbb{R}, \cdot)$ is a cocycle and in this case, $\theta(x, y)$ is represented by $e^{B(x, y)} e^{M(x y)-M(x)-M(y)}$.

Let us consider the generalized characterization of a symmetrically compositive sum form related to distance measures with a cocycle:

$(C D M) f(p r, q s)+f(p s, q r)=\theta(p q, r s) f(p, q) f(r, s)$

for all $p, q, r, s \in G$ and where $f, \theta$ are functionals on $G^{2}$, which can be represented as exponential functional equation in reduction. 
In fact, if $f(x, y)=\frac{1}{x}+\frac{1}{y}$, then $f(p r, q s)+f(p s, q r)=f(p, q) f(r, s)$, and also if $f(x, y)=a^{\ln x y}$, and $\theta(x, y)=2$ then $f, \theta$ satisfy the equation $f(p r, q s)+f(p s, q r)=\theta(p q, r s) f(p, q) f(r, s)$.

This paper aims to investigate the superstability of four generalized functional equations of $(C D M)$, namely, as well as that of the following type functional equations:

$\left(G M_{f f f g}\right) f(p r, q s)+f(p s, q r)=\theta(p q, r s) f(p, q) g(r, s)$,

$\left(G M_{f f g f}\right) f(p r, q s)+f(p s, q r)=\theta(p q, r s) g(p, q) f(r, s)$,

$\left(G M_{f f g g}\right) f(p r, q s)+f(p s, q r)=\theta(p q, r s) g(p, q) g(r, s)$,

$\left(G M_{f g h}\right) f(p r, q s)+f(p s, q r)=\theta(p q, r s) g(p, q) h(r, s)$.

\section{Superstability of the equations}

In this section, we investigate the superstability of $(C D M)$ and four generalized functional equations $\left(G M_{f f g}\right),\left(G M_{f f g}\right),\left(G M_{f f g}\right)$, and $\left(G M_{f g h}\right)$.

Theorem 1 Let $f, g: G^{2} \rightarrow \mathbb{R}, \phi: G^{2} \rightarrow \mathbb{R}_{+}$be functions and a function $\theta: G^{2} \rightarrow \mathbb{R}_{k}$ be a cocycle satisfying

$$
|f(p r, q s)+f(p s, q r)-\theta(p q, r s) g(p, q) h(r, s)| \leq \phi(r, s) \quad \forall p, q, r, s \in G .
$$

and $|f(p, q)-g(p, q)| \leq M$ for all $p, q \in G$ and some constant $M$.

Then either $g$ is bounded or $h$ satisfies $(C D M)$.

Proof Let $g$ be an unbounded solution of inequality (2.1). Then there exists a sequence $\left\{\left(x_{n}, y_{n}\right) \mid n \in N\right\}$ in $G^{2}$ such that $0 \neq\left|g\left(x_{n}, y_{n}\right)\right| \rightarrow \infty$ as $n \rightarrow \infty$.

Letting $p=x_{n}, q=y_{n}$ in (2.1) and dividing by $\left|\theta\left(x_{n} y_{n}, r s\right) g\left(x_{n}, y_{n}\right)\right|$, we have

$$
\left|\frac{f\left(x_{n} r, y_{n} s\right)+f\left(x_{n} s, y_{n} r\right)}{\theta\left(x_{n} y_{n}, r s\right) g\left(x_{n}, y_{n}\right)}-h(r, s)\right| \leq \frac{\phi(r, s)}{k\left|g\left(x_{n}, y_{n}\right)\right|} .
$$

Passing to the limit as $n \rightarrow \infty$, we obtain

$$
h(r, s)=\lim _{n \rightarrow \infty} \frac{f\left(x_{n} r, y_{n} s\right)+f\left(x_{n} s, y_{n} r\right)}{\theta\left(x_{n} y_{n}, r s\right) g\left(x_{n}, y_{n}\right)} .
$$

Letting $p=x_{n} p, q=y_{n} q$ in (2.1) and dividing by $\left|g\left(x_{n}, y_{n}\right)\right|$, we have

$$
\begin{aligned}
& \left|\frac{f\left(x_{n} p r, y_{n} q s\right)+f\left(x_{n} p s, y_{n} q r\right)}{g\left(x_{n}, y_{n}\right)}-\frac{\theta\left(x_{n} p y_{n} q, r s\right) g\left(x_{n} p, y_{n} q\right)}{g\left(x_{n}, y_{n}\right)} h(r, s)\right| \\
& \quad \leq \frac{\phi(r, s)}{\left|g\left(x_{n}, y_{n}\right)\right|} \rightarrow 0
\end{aligned}
$$

as $n \rightarrow \infty$.

Letting $p=x_{n} q, q=y_{n} p$ in (2.1) and dividing by $\left|g\left(x_{n}, y_{n}\right)\right|$, we have

$$
\begin{aligned}
& \left|\frac{f\left(x_{n} q r, y_{n} p s\right)+f\left(x_{n} q s, y_{n} p r\right)}{g\left(x_{n}, y_{n}\right)}-\frac{\theta\left(x_{n} q y_{n} p, r s\right) g\left(x_{n} q, y_{n} p\right)}{g\left(x_{n}, y_{n}\right)} h(r, s)\right| \\
& \quad \leq \frac{\phi(r, s)}{\left|g\left(x_{n}, y_{n}\right)\right|} \rightarrow 0
\end{aligned}
$$

as $n \rightarrow \infty$ 
Note that for any $a, b, c$ in $G, \theta(b a, c) \theta(b, a)=\theta(b, a c) \theta(a, c)$ by the definition of the cocycle. Letting $p q=a, x_{n} y_{n}=b$, and $r s=c$ we have

$$
\frac{\theta\left(x_{n} y_{n} p q, r s\right) \theta\left(x_{n} y_{n}, p q\right)}{\theta\left(x_{n} y_{n}, p q r s\right)}=\theta(p q, r s)
$$

for any $p, q, r, s, x_{n}, y_{n}$ in G. Thus, from (2.2), (2.3), and (2.4), we obtain

$$
\begin{aligned}
& |h(p r, q s)+h(p s, q r)-\theta(p q, r s) h(p, q) h(r, s)| \\
& =\lim _{n \rightarrow \infty} \mid \frac{f\left(x_{n} p r, y_{n} q s\right)+f\left(x_{n} q s, y_{n} p r\right)+f\left(x_{n} p s, y_{n} q r\right)+f\left(x_{n} q r, y_{n} p s\right)}{\theta\left(x_{n} y_{n}, p r q s\right) g\left(x_{n}, y_{n}\right)} \\
& -\theta(p q, r s) h(p, q) h(r, s) \\
& \leq \lim _{n \rightarrow \infty}\left|\frac{1}{\theta\left(x_{n} y_{n}, p r q s\right)}\right| \cdot \mid \frac{f\left(x_{n} p r, y_{n} q s\right)+f\left(x_{n} p s, y_{n} q r\right)}{g\left(x_{n}, y_{n}\right)} \\
& -\frac{\theta\left(x_{n} p y_{n} q, r s\right) g\left(x_{n} p, y_{n} q\right) h(r, s)}{g\left(x_{n}, y_{n}\right)} \mid \\
& +\lim _{n \rightarrow \infty}\left|\frac{1}{\theta\left(x_{n} y_{n}, p r q s\right)}\right| \cdot \mid \frac{f\left(x_{n} q r, y_{n} p s\right)+f\left(x_{n} q s, y_{n} p r\right)}{g\left(x_{n}, y_{n}\right)} \\
& -\frac{\theta\left(x_{n} q y_{n} p, r s\right) g\left(x_{n} q, y_{n} p\right) h(r, s)}{g\left(x_{n}, y_{n}\right)} \mid \\
& +|h(r, s)| \lim _{n \rightarrow \infty} \mid \frac{\theta\left(x_{n} y_{n} p q, r s\right) \theta\left(x_{n} y_{n}, p q\right)}{\theta\left(x_{n} y_{n}, p q r s\right)} \cdot \frac{g\left(x_{n} p, y_{n} q\right)+g\left(x_{n} q, y_{n} p\right)}{\theta\left(x_{n} y_{n}, p q\right) g\left(x_{n}, y_{n}\right)} \\
& -\theta(p q, r s) h(p, q) \\
& \leq h(r, s) \theta(p q, r s) \lim _{n \rightarrow \infty} \mid \frac{f\left(x_{n} p, y_{n} q\right)+f\left(x_{n} q, y_{n} p\right)}{\theta\left(x_{n} y_{n}, p q\right) g\left(x_{n}, y_{n}\right)} \\
& +\frac{(g-f)\left(x_{n} p, y_{n} q\right)+(g-f)\left(x_{n} q, y_{n} p\right)}{\theta\left(x_{n} y_{n}, p q\right) g\left(x_{n}, y_{n}\right)}-h(p, q) \mid \\
& \leq h(r, s) \theta(p q, r s) \lim _{n \rightarrow \infty}\left|\frac{2 M}{k g\left(x_{n}, y_{n}\right)}\right| \\
& +h(r, s) \theta(p q, r s) \lim _{n \rightarrow \infty}\left|\frac{f\left(x_{n} p, y_{n} q\right)+f\left(x_{n} q, y_{n} p\right)}{\theta\left(x_{n} y_{n}, p q\right) g\left(x_{n}, y_{n}\right)}-h(p, q)\right| \\
& =0 \text {. }
\end{aligned}
$$

Theorem 2 Let $f, g: G^{2} \rightarrow \mathbb{R}, \phi: G^{2} \rightarrow \mathbb{R}_{+}$be functions and a function $\theta: G^{2} \rightarrow \mathbb{R}_{k}$ be a cocycle satisfying

$$
|f(p r, q s)+f(p s, q r)-\theta(p q, r s) g(p, q) h(r, s)| \leq \phi(p, q) \quad \forall p, q, r, s \in G
$$

and $|f(p, q)-h(p, q)| \leq M$ for all $p, q \in G$ and some constant $M$.

Then either $h$ is bounded or $g$ satisfies (CDM).

Proof For $h$ to be an unbounded solution of inequality (2.5), we can choose a sequence $\left\{\left(x_{n}, y_{n}\right) \mid n \in N\right\}$ in $G^{2}$ such that $0 \neq\left|h\left(x_{n}, y_{n}\right)\right| \rightarrow \infty$ as $n \rightarrow \infty$. 
Letting $r=x_{n}, s=y_{n}$ in (2.5) and dividing by $\left|\theta\left(p q, x_{n} y_{n}\right) h\left(x_{n}, y_{n}\right)\right|$, we have

$$
\left|\frac{f\left(p x_{n}, q y_{n}\right)+f\left(p y_{n}, q x_{n}\right)}{\theta\left(p q, x_{n} y_{n}\right) h\left(x_{n}, y_{n}\right)}-g(p, q)\right| \leq \frac{\phi(p, q)}{k\left|h\left(x_{n}, y_{n}\right)\right|}
$$

Passing to the limit as $n \rightarrow \infty$, we obtain

$$
g(p, q)=\lim _{n \rightarrow \infty} \frac{f\left(p x_{n}, q y_{n}\right)+f\left(p y_{n}, q x_{n}\right)}{\theta\left(p q, x_{n} y_{n}\right) h\left(x_{n}, y_{n}\right)}
$$

Replacing $r=r x_{n}, s=s y_{n}$ in (2.5) and dividing by $\left|h\left(x_{n}, y_{n}\right)\right|$, we have

$$
\begin{aligned}
& \left|\frac{f\left(p r x_{n}, q s y_{n}\right)+f\left(p s y_{n}, q r x_{n}\right)}{h\left(x_{n}, y_{n}\right)}-\theta\left(p q, r x_{n} s y_{n}\right) g(p, q) \frac{h\left(r x_{n}, s y_{n}\right)}{h\left(x_{n}, y_{n}\right)}\right| \\
& \quad \leq \frac{\phi(p, q)}{\left|h\left(x_{n}, y_{n}\right)\right|} \rightarrow 0
\end{aligned}
$$

as $n \rightarrow \infty$.

Replacing $r=r y_{n}, s=s x_{n}$ in (2.5) and dividing by $\left|h\left(x_{n}, y_{n}\right)\right|$, we have

$$
\begin{aligned}
& \left|\frac{f\left(p r y_{n}, q s x_{n}\right)+f\left(p s x_{n}, q r y_{n}\right)}{h\left(x_{n}, y_{n}\right)}-g(p, q) \theta\left(p q, r y_{n} s x_{n}\right) \frac{h\left(r y_{n}, s x_{n}\right)}{h\left(x_{n}, y_{n}\right)}\right| \\
& \leq \frac{\phi(p, q)}{\left|h\left(x_{n}, y_{n}\right)\right|} \rightarrow 0
\end{aligned}
$$

as $n \rightarrow \infty$.

Thus from (2.6), (2.7), and (2.8), we obtain

$$
\begin{aligned}
|g(p r, q s)+g(p s, q r)-\theta(p q, r s) g(p, q) g(r, s)| \\
=\lim _{n \rightarrow \infty} \mid \frac{f\left(p r x_{n}, q s y_{n}\right)+f\left(p r y_{n}, q s x_{n}\right)+f\left(p s x_{n}, q r y_{n}\right)+f\left(p s y_{n}, q r x_{n}\right)}{\theta\left(p r q s, x_{n} y_{n}\right) h\left(x_{n}, y_{n}\right)} \\
\quad-\theta(p q, r s) g(p, q) g(r, s) \mid \\
\leq \lim _{n \rightarrow \infty}\left|\frac{1}{\theta\left(p q r s, x_{n} y_{n}\right)}\right| \cdot \mid \frac{f\left(p r x_{n}, q s y_{n}\right)+f\left(p s y_{n}, q r x_{n}\right)}{h\left(x_{n}, y_{n}\right)} \\
\quad-g(p, q) \theta\left(p q, r x_{n} s y_{n}\right) \frac{h\left(r x_{n}, s y_{n}\right)}{h\left(x_{n}, y_{n}\right)} \mid \\
\quad+\lim _{n \rightarrow \infty}\left|\frac{1}{\theta\left(p q r s, x_{n} y_{n}\right)}\right| \cdot \mid \frac{f\left(p r y_{n}, q s x_{n}\right)+f\left(p s x_{n}, q r y_{n}\right)}{h\left(x_{n}, y_{n}\right)} \\
\quad-g(p, q) \theta\left(p q, r y_{n} s x_{n}\right) \frac{h\left(r y_{n}, s x_{n}\right)}{h\left(x_{n}, y_{n}\right)} \mid \\
\quad+|g(p, q)| \lim _{n \rightarrow \infty} \mid \frac{\theta\left(p q, r x_{n} s y_{n}\right) \theta\left(r s, x_{n} y_{n}\right)}{\theta\left(p q r s, x_{n} y_{n}\right)} \cdot \frac{h\left(r x_{n}, s y_{n}\right)+h\left(r y_{n}, s x_{n}\right)}{\theta\left(r s, x_{n} y_{n}\right) h\left(x_{n} y_{n}\right)} \\
\quad-\theta(p q, r s) g(r, s) \mid \\
=|g(p, q)| \theta(p q, r s) \lim _{n \rightarrow \infty} \mid \frac{(h-f)\left(r x_{n}, s y_{n}\right)+(h-f)\left(r y_{n}, s x_{n}\right)}{\theta\left(r s, x_{n} y_{n}\right) h\left(x_{n}, y_{n}\right)} \\
\end{aligned}
$$




$$
\begin{aligned}
& +\frac{f\left(r x_{n}, s y_{n}\right)+f\left(r y_{n}, s x_{n}\right)}{\theta\left(r s, x_{n} y_{n}\right) h\left(x_{n}, y_{n}\right)}-g(r, s) \mid \\
\leq & |g(p, q)| \theta(p q, r s) \frac{2 M}{k\left|h\left(x_{n}, y_{n}\right)\right|} \\
& +|g(p, q)| \theta(p q, r s) \lim _{n \rightarrow \infty}\left|\frac{f\left(r x_{n}, s y_{n}\right)+f\left(r y_{n}, s x_{n}\right)}{\theta\left(r s, x_{n} y_{n}\right) h\left(x_{n}, y_{n}\right)}-g(r, s)\right| \\
= & 0 .
\end{aligned}
$$

Corollary 1 Let $f, g: G^{2} \rightarrow \mathbb{R}, \phi: G^{2} \rightarrow \mathbb{R}_{+}$be functions and a function $\theta: G^{2} \rightarrow \mathbb{R}_{k}$ be a cocycle satisfying

$$
|f(p r, q s)+f(p s, q r)-\theta(p q, r s) g(p, q) g(r, s)| \leq \phi(p, q) \text { or } \phi(r, s)
$$

for any $p, q, r, s \in G$ and $|f(p, q)-g(p, q)| \leq M$ for all $p, q \in G$ and some constant $M$. Then either $g$ is bounded or $g$ satisfies (CDM).

Corollary 2 Let $f, g: G^{2} \rightarrow \mathbb{R}, \phi: G^{2} \rightarrow \mathbb{R}_{+}$be functions and a function $\theta: G^{2} \rightarrow \mathbb{R}_{k}$ be a cocycle satisfying

$$
|f(p r, q s)+f(p s, q r)-\theta(p q, r s) f(p, q) g(r, s)| \leq \phi(p, q)
$$

for any $p, q, r, s \in G$. Then either $g$ is bounded, or $f$ satisfies (CDM) and also $f$ and $g$ satisfy $\left(G M_{f f g}\right)$.

Corollary 3 Let $f, g: G^{2} \rightarrow \mathbb{R}, \phi: G^{2} \rightarrow \mathbb{R}_{+}$be functions and a function $\theta: G^{2} \rightarrow \mathbb{R}_{k}$ be a cocycle satisfying

$$
|f(p r, q s)+f(p s, q r)-\theta(p q, r s) f(p, q) g(r, s)| \leq \phi(r, s)
$$

for any $p, q, r, s \in G$. Then either $f$ is bounded, or $g$ satisfies (CDM) and also $g$ and $f$ satisfy $\left(G M_{g g g f}\right) g(p r, q s)+g(p s, q r)-\theta(p q, r s) g(p, q) f(r, s)$.

Corollary 4 Let $f, g: G^{2} \rightarrow \mathbb{R}, \phi: G^{2} \rightarrow \mathbb{R}_{+}$be functions and a function $\theta: G^{2} \rightarrow \mathbb{R}_{k}$ be a cocycle satisfying

$$
|f(p r, q s)+f(p s, q r)-\theta(p q, r s) g(p, q) f(r, s)| \leq \phi(p, q) \quad \forall p, q, r, s \in G
$$

for any $p, q, r, s \in G$. Then either $f$ is bounded, or $g$ satisfies (CDM) and also $f$ and $g$ satisfy $\left(G M_{g g g f}\right)$.

Corollary 5 Let $f, g: G^{2} \rightarrow \mathbb{R}, \phi: G^{2} \rightarrow \mathbb{R}_{+}$be functions and a function $\theta: G^{2} \rightarrow \mathbb{R}_{k}$ be a cocycle satisfying

$$
|f(p r, q s)+f(p s, q r)-\theta(p q, r s) g(p, q) f(r, s)| \leq \phi(r, s) \quad \forall p, q, r, s \in G
$$

for any $p, q, r, s \in G$. Then either $g$ is bounded, or $f$ satisfies (CDM) and also $f$ and $g$ satisfy $\left(G M_{f f g}\right)$. 
Corollary 6 Let $f, g: G^{2} \rightarrow \mathbb{R}, \phi: G^{2} \rightarrow \mathbb{R}_{+}$be functions and a function $\theta: G^{2} \rightarrow \mathbb{R}_{k}$ be a cocycle satisfying

$$
|f(p r, q s)+f(p s, q r)-\theta(p q, r s) g(p, q) f(r, s)| \leq \phi(p, q) \quad \forall p, q, r, s \in G
$$

for any $p, q, r, s \in G$. Then either $f$ is bounded, or $g$ satisfies $(C D M)$ and also $f$ and $g$ satisfy $\left(G M_{g g g f}\right)$.

Corollary 7 Let $k>0$ and $f, g: G^{2} \rightarrow \mathbb{R}, \phi: G^{2} \rightarrow \mathbb{R}_{+}$be functions satisfying

$$
\left|f(p r, q s)+f(p s, q r)-k^{\ln (p q) \ln (r s)} f(p, q) f(r, s)\right| \leq \phi(p, q) \text { or } \phi(r, s)
$$

for any $p, q, r, s \in G$. Then either $f$ is bounded or $f$ satisfies the following equation:

$$
f(p r, q s)+f(p s, q r)=k^{\ln (p q) \ln (r s)} f(p, q) f(r, s) .
$$

Corollary 8 Letf, $g: G^{2} \rightarrow \mathbb{R}, \phi: G^{2} \rightarrow \mathbb{R}_{+}$be functions satisfying

$$
|f(p r, q s)+f(p s, q r)-f(p, q) f(r, s)| \leq \phi(p, q) \text { or } \phi(r, s)
$$

for any $p, q, r, s \in G$. Then either $f$ is bounded or $f$ satisfies $(F E)$.

Theorem 3 Let $f, g: G^{2} \rightarrow \mathbb{R}, \phi: G^{2} \rightarrow \mathbb{R}_{+}$be functions and a function $\theta: G^{2} \rightarrow \mathbb{R}_{k}$ be a cocycle satisfying

$$
|f(p r, q s)+f(p s, q r)-\theta(p q, r s) f(p, q) g(r, s)| \leq \varepsilon
$$

for any $p, q, r, s \in G$. Then $f$ (or $g$ ) is bounded, or $f$ and $g$ satisfy (CDM) and also $f, g, \theta$ satisfy $\left(G M_{f f f}\right)$.

Proof Replacing $g(p, q)$ by $f(p, q)$ and $h(r, s)$ by $g(r, s)$ for all $p, q, r, s \in G$ in Theorem 1 , we find that $f$ is bounded or $g$ satisfies $(C D M)$. Note that $f$ is bounded iff $g$ is bounded. Namely, for all $p, q, r, s \in G$

$$
|g(r, s)| \leq \frac{\varepsilon+f(p r, q s)+f(p s, q r)}{k|f(p, q)|} .
$$

Let $g$ be unbounded. Then $f$ is unbounded by a similar method to the proof of Theorem $1 ; g$ satisfies $(C D M)$. Now by a similar method to the calculation in Theorem 1 with the unboundedness of $g$, we have

$$
f(p, q)=\lim _{n \rightarrow \infty} \frac{f\left(p x_{n}, q y_{n}\right)+f\left(p y_{n}, q x_{n}\right)}{\theta\left(p q, x_{n} y_{n}\right) g\left(x_{n}, y_{n}\right)}
$$

for any $r, s, x_{n}, y_{n} \in G$. Since $g$ satisfies $(C D M)$, we have

$$
\begin{aligned}
& |f(p r, q s)+f(p s, q r)-\theta(p q, r s) f(p, q) g(r, s)| \\
& \quad=\lim _{n \rightarrow \infty} \mid \frac{f\left(p r x_{n}, q s y_{n}\right)+f\left(p r y_{n}, q s x_{n}\right)+f\left(p s x_{n}, q r y_{n}\right)+f\left(p s y_{n}, q r x_{n}\right)}{\theta\left(p r q s, x_{n} y_{n}\right) g\left(x_{n}, y_{n}\right)}
\end{aligned}
$$




$$
\begin{aligned}
& -\theta(p q, r s) f(p, q) g(r, s) \mid \\
\leq & \lim _{n \rightarrow \infty}\left|\frac{1}{\theta\left(p q r s, x_{n} y_{n}\right)}\right| \cdot \mid \frac{f\left(p r x_{n}, q s y_{n}\right)+f\left(p s y_{n}, q r x_{n}\right)}{g\left(x_{n}, y_{n}\right)} \\
& -f(p, q) \theta\left(p q, r x_{n} s y_{n}\right) \frac{g\left(r x_{n}, s y_{n}\right)}{g\left(x_{n}, y_{n}\right)} \mid \\
& +\lim _{n \rightarrow \infty}\left|\frac{1}{\theta\left(p q r s, x_{n} y_{n}\right)}\right| \cdot \mid \frac{f\left(p r y_{n}, q s x_{n}\right)+f\left(p s x_{n}, q r y_{n}\right)}{g\left(x_{n}, y_{n}\right)} \\
& -f(p, q) \theta\left(p q, r y_{n} s x_{n}\right) \frac{g\left(r y_{n}, s x_{n}\right)}{g\left(x_{n}, y_{n}\right)} \mid \\
& +|f(p, q)| \lim _{n \rightarrow \infty} \mid \frac{\theta\left(p q, r x_{n} s y_{n}\right) \theta\left(r s, x_{n} y_{n}\right)}{\theta\left(p q r s, x_{n} y_{n}\right)} \cdot \frac{g\left(r x_{n}, s y_{n}\right)+g\left(r y_{n}, s x_{n}\right)}{\theta\left(r s, x_{n} y_{n}\right) g\left(x_{n} y_{n}\right)} \\
& -\theta(p q, r s) g(r, s) \mid \\
= & |f(p, q)| \lim _{n \rightarrow \infty} \mid \frac{\theta\left(p q, r x_{n} s y_{n}\right) \theta\left(r s, x_{n} y_{n}\right)}{\theta\left(p q r s, x_{n} y_{n}\right)} \cdot \frac{g\left(r x_{n}, s y_{n}\right)+g\left(r y_{n}, s x_{n}\right)}{\theta\left(r s, x_{n} y_{n}\right) g\left(x_{n} y_{n}\right)} \\
= & |f(p, q)||\theta(p q, r s) g(r, s)-\theta(p q, r s) g(r, s)|=0 .
\end{aligned}
$$

Thus $f$ and $g$ imply the required $\left(G M_{f f f g}\right)$. The same procedure implies that the above inequalities change to

$$
\begin{aligned}
& |f(p r, q s)+f(p s, q r)-\theta(p q, r s) f(p, q) f(r, s)| \\
& \quad \leq|f(p, q)| \lim _{n \rightarrow \infty}\left|\frac{\theta\left(p q, r x_{n} s y_{n}\right) \theta\left(r s, x_{n} y_{n}\right)}{\theta\left(p q r s, x_{n} y_{n}\right)} \cdot \frac{f\left(r x_{n}, s y_{n}\right)+f\left(r y_{n}, s x_{n}\right)}{\theta\left(r s, x_{n} y_{n}\right) g\left(x_{n} y_{n}\right)}-\theta(p q, r s) f(r, s)\right| \\
& \quad=|f(p, q)||\theta(p q, r s) f(r, s)-\theta(p q, r s) f(r, s)|=0,
\end{aligned}
$$

as desired.

The proof of the following theorem is the same procedure as in the proof of Theorem 3.

Theorem 4 Let $f, g: G^{2} \rightarrow \mathbb{R}, \phi: G^{2} \rightarrow \mathbb{R}_{+}$be functions and a function $\theta: G^{2} \rightarrow \mathbb{R}_{k}$ be a cocycle satisfying

$$
|f(p r, q s)+f(p s, q r)-\theta(p q, r s) g(p, q) f(r, s)| \leq \varepsilon
$$

for any $p, q, r, s \in G$. Then $f$ (or $g$ ) is bounded, or $f$ and $g$ satisfy (CDM) and also $f, g, \theta$ satisfy $\left(G M_{f f f}\right)$.

\section{Example 1 Let}

$$
f(x, y)=a^{\ln x y}+\frac{\varepsilon}{2}, \quad g(x, y)=a^{\ln x y}, \quad \theta(x, y)=2
$$


Then we have

$$
|f(p, q)-g(p, q)| \leq \frac{\varepsilon}{2}
$$

and

$$
\begin{aligned}
& |f(p r, q s)+f(p s, q r)-\theta(p q, r s) g(p, q) g(r, s)| \\
& \quad=\left|a^{\ln p r q s}+a^{\ln p s q r}+\varepsilon-2 a^{\ln p q} a^{\ln r s}\right| \\
& \quad=\varepsilon .
\end{aligned}
$$

Thus $g$ satisfies $(C D M)$. But $f, g, \theta$ being nonzero functions do not satisfy $\left(G M_{f f g}\right)$.

Let $(S ; \diamond)$ and $(\widetilde{S} ; \diamond)$ be a semigroup and a group with semigroup operation $\diamond$, respectively.

Theorem 5 Let $f, g, h: S^{2}, \widetilde{S}^{2} \rightarrow \mathbb{R}$ and $\phi: S^{2}, \widetilde{S}^{2} \rightarrow \mathbb{R}$ be a nonzero function satisfying

$$
\begin{aligned}
& |f(p \diamond r, q \diamond s)+f(p \diamond s, q \diamond r)-\theta(p q, r s) f(p, q) g(r, s)| \\
& \quad \leq\left\{\begin{array}{rrr}
\text { (i) } & \phi(r, s) & \forall p, q, r, s \in \widetilde{S}, \\
\text { (ii) } & \phi(p, q) & \forall p, q, r, s \in S .
\end{array}\right.
\end{aligned}
$$

(a) In case (i), let $|f(p, q)-g(p, q)| \leq M$ for all $p, q \in S$ and some constant $M$.

Then either $g$ is bounded or $h$ satisfies $(C D M)$.

(b) In case (ii), let $|f(p, q)-h(p, q)| \leq M$ for all $p, q \in G$ and some constant $M$.

Then either $h$ is bounded or $g$ satisfies (CDM).

\section{Competing interests}

The authors declare that they have no competing interests.

\section{Authors' contributions}

All authors contributed equally to the writing of this paper. All authors read and approved the final manuscript.

\section{Author details}

'Department of Computer Hacking and Information Security, College of Natural Science, Daejeon University, Daejeon, 300-716, Republic of Korea. ${ }^{2}$ Department of Mathematics, Kangnam University, Yongin, Gyeonggi 446-702, Korea.

\section{Acknowledgements}

The first author and the second author of this work were supported by Basic Science Research Program through the National Research Foundation of Korea (NRF) funded by the Ministry of Education, Science and Technology (Grant No. 2013-0154000) and (Grant number: 2010-0010243), respectively.

Received: 23 April 2014 Accepted: 29 September 2014 Published: 15 Oct 2014

\section{References}

1. Chung, JK, Kannappan, PI, Ng, CT, Sahoo, PK: Measures of distance between probability distributions. J. Math. Anal. Appl. 138, 280-292 (1989)

2. Sahoo, PK: On a functional equation associated with stochastic distance measures. Bull. Korean Math. Soc. 36 , 287-303 (1999)

3. Kim, GH, Sahoo, PK: Stability of a functional equation related to distance measure - I. Appl. Math. Lett. 24, 843-849 (2011)

4. Kim, GH, Sahoo, PK: Stability of a functional equation related to distance measure - II. Ann. Funct. Anal. 1, $26-35$ (2010)

5. Kannappan, PI, Sahoo, PK: Sum form distance measures between probability distributions and functional equations. Int. J. Math. Stat. Sci. 6, 91-105 (1997)

6. Kannappan, PI, Sahoo, PK, Chung, JK: On a functional equation associated with the symmetric divergence measures. Util. Math. 44, 75-83 (1993) 
7. Riedel, T, Sahoo, PK: On a generalization of a functional equation associated with the distance between the probability distributions. Publ. Math. (Debr.) 46, 125-135 (1995)

8. Riedel, T, Sahoo, PK: On two functional equations connected with the characterizations of the distance measures. Aequ. Math. 54, 242-263 (1998)

9. Riedel, T, Sahoo, PK: On a generalization of a functional equation associated with the distance between the probability distributions. Publ. Math. (Debr.) 46, 125-135 (1995)

10. Tabor, J: Hyers theorem and the cocycle property. In: Daróczy, Z, Páles, Z (eds.) Functional Equations-Results and Advances, pp. 275-290. Kluwer Academic, Dordrecht (2002)

11. Hosszú, M: On the functional equation $f(x+y, z)+f(x, y)=f(x, y+z)+f(y, z)$. Period. Math. Hung. 1(3), $213-216(1971)$

10.1186/1029-242X-2014-393

Cite this article as: Lee and Kim: Superstability of the functional equation with a cocycle related to distance measures. Journal of Inequalities and Applications 2014, 2014:393

\section{Submit your manuscript to a SpringerOpen ${ }^{\circ}$ journal and benefit from:}

- Convenient online submission

- Rigorous peer review

- Immediate publication on acceptance

- Open access: articles freely available online

- High visibility within the field

- Retaining the copyright to your article 